\title{
Optimizing Cerebral Autoregulation May Decrease Neonatal Regional Hypoxic-Ischemic Brain Injury
}

\author{
Jennifer K. Lee ${ }^{a, b} \quad$ Andrea Poretti ${ }^{b, c}$ Jamie Perin ${ }^{d}$ Thierry A.G.M. Huisman ${ }^{b, c}$ \\ Charlamaine Parkinson $^{\text {b, e }}$ Raul Chavez-Valdez ${ }^{\text {b, e }}$ Matthew O'Connor ${ }^{\text {b, e }}$ \\ Michael Reyes $^{a} \quad$ Jillian Armstrong ${ }^{a}$ Jacky M. Jennings ${ }^{d}$ Maureen M. Gilmore ${ }^{b}$ e \\ Raymond C. Koehler $^{\mathrm{a}}$ Frances J. Northington $^{\mathrm{b}, \mathrm{e}}$ Aylin Tekes $^{\mathrm{b}, \mathrm{c}}$ \begin{abstract}
Pediatric Radiology and Pediatric Neuroradiology, Department of Radiology, ${ }^{\mathrm{d} C e n t e r}$ for Child and Community Health Research (CCHR), and e Division of Neonatology, Department of Pediatrics, Johns Hopkins School of
\end{abstract} \\ ${ }^{a}$ Department of Anesthesiology and Critical Care Medicine, ${ }^{b}$ Neurosciences Intensive Care Nursery, ${ }^{c}$ Division of \\ Medicine, Baltimore, MD, USA
}

\section{Keywords}

Brain injury - Cerebral blood flow $\cdot$ Hypoxia

Hypoxic-ischemic encephalopathy $\cdot$ Neonates

\begin{abstract}
Background: Therapeutic hypothermia provides incomplete neuroprotection for neonatal hypoxic-ischemic encephalopathy (HIE). We examined whether hemodynamic goals that support autoregulation are associated with decreased brain injury and whether these relationships are affected by birth asphyxia or vary by anatomic region. Methods: Neonates cooled for HIE received near-infrared spectroscopy autoregulation monitoring to identify the mean arterial blood pressure with optimized autoregulatory function (MAP ${ }_{\mathrm{OPT}}$ ). Blood pressure deviation from MAP $\mathrm{PPT}_{\mathrm{OPT}}$ was correlated with brain injury on MRI after adjusting for the effects of arterial carbon dioxide, vasopressors, seizures,
\end{abstract}

and birth asphyxia severity. Results: Blood pressure deviation from MAP ${ }_{\mathrm{OPT}}$ related to neurologic injury in several regions independent of birth asphyxia severity. Greater duration and deviation of blood pressure below MAP $\mathrm{PPT}_{\mathrm{OPT}}$ were associated with greater injury in the paracentral gyri and white matter. Blood pressure within $\mathrm{MAP}_{\mathrm{OPT}}$ related to lesser injury in the white matter, putamen and globus pallidus, and brain stem. Finally, blood pressures that exceeded

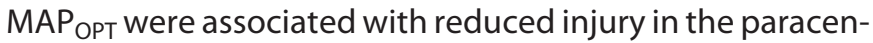
tral gyri. Conclusions: Blood pressure deviation from optimal autoregulatory vasoreactivity was associated with MRI markers of brain injury that, in many regions, were independent of the initial birth asphyxia. Targeting hemodynamic ranges to optimize autoregulation has potential as an adjunctive therapy to hypothermia for HIE.

(c) 2016 S. Karger AG, Basel

\section{KARGER}

(C) 2016 S. Karger AG, Basel

E-Mail karger@karger.com

www.karger.com/dne
Jennifer K. Lee, MD

Department of Anesthesiology and Critical Care Medicine Johns Hopkins University, 1800 Orleans Street, Room 6321

Baltimore, MD 21287 (USA)

E-Mail jklee@jhmi.edu 


\section{Introduction}

Therapeutic hypothermia offers only partial neuroprotection for neonates with hypoxic-ischemic encephalopathy (HIE). Even with hypothermia, neurologic disabilities persist in $35-55 \%$ of survivors at $6-7$ years $[1,2]$. Ensuring robust cerebrovascular autoregulation might improve outcomes. In pilot studies, we showed that blood pressure deviations from the optimal mean arterial blood pressure $\left(\mathrm{MAP}_{\mathrm{OPT}}\right)$ at which autoregulation is most robust are associated with brain injury on MRI $[3,4]$ and 2-year neurodevelopmental outcomes [5] after HIE. However, these studies were too small to control for confounders that affect autoregulation, such as the arterial partial pressure of carbon dioxide $\left(\mathrm{PaCO}_{2}\right)$ [6] and vasopressors $[7,8]$. Here, we studied a larger cohort to evaluate autoregulation independent of $\mathrm{PaCO}_{2}$, vasopressors, perinatal insult severity, and seizures.

Autoregulation can be measured indirectly with nearinfrared spectroscopy (NIRS) [3-5, 9, 10]. NIRS measures deoxy- and oxyhemoglobin optical densities from the regional cortex, and the sum of these densities - the relative total tissue hemoglobin $(\mathrm{rTHb})$ - is a surrogate measure of regional cerebral blood volume. NIRS rTHb measurements reflect the fluctuations in cerebral blood volume that occur during autoregulatory vasoactive responses to changes in arterial blood pressure. The hemoglobin volume index $(\mathrm{HVx})$ is calculated as the correlation coefficient between $\mathrm{rTHb}$ and MAP [11].

Because NIRS interrogation focuses on the frontal cortex, whether autoregulation measurements can gauge injury risk in other anatomic regions is unclear and must be studied to determine the relevance for HIE. Our objective was to measure autoregulation with HVx and brain injury with MRI in neonates who received therapeutic hypothermia for HIE to test the hypotheses that (1) HVx identifies the blood pressure range with optimal autoregulatory vasoreactivity; (2) autoregulation affects brain injury independent of perinatal insult severity; and (3) these relationships vary by anatomic region.

\section{Methods}

This observational, prospective study was approved by the Johns Hopkins University School of Medicine Institutional Review Board. Written informed consents were obtained until May 2013, when NIRS became standard of care for HIE treatment at our hospital. After that, we were granted a waiver of consent. We sequentially screened all neonates admitted for HIE between September 2010 and July 2015 using HIE diagnostic criteria from the NICHD
Neonatal Research Network's trial of therapeutic hypothermia in HIE [12] and the eligibility criteria from our prior studies [3-5]. The neonates reported here include those from our pilot studies [3-5] with an additional 36 neonates. We excluded neonates who received extracorporeal membrane support (ECMO).

\section{Clinical Care}

Neonates received therapeutic hypothermia for $72 \mathrm{~h}$ per previously described protocols [3-5]. We examined HVx during hypothermia, rewarming, and the first $6 \mathrm{~h}$ of normothermia. Clinicians determined the neonates' blood pressure goals and could view the NIRS regional cerebral oximetry $\left(\mathrm{rSO}_{2}\right)$ but were blinded to $\mathrm{HVx}$. When necessary, dopamine was initiated followed by dobutamine and epinephrine. Seizures were diagnosed by electroencephalogram and treated with phenobarbital. Hydrocortisone was initiated for adrenal suppression or persistent hypotension refractory to vasopressors when needed.

Clinical data were obtained from the medical record by investigators (R.C.-V. and M.O'C.) blinded to the autoregulation and brain MRI data. $\mathrm{PaCO}_{2}$ levels were classified into four categories [13]: (1) all $\mathrm{PaCO}_{2}$ levels 35-45 mm Hg; (2) some $<35 \mathrm{~mm} \mathrm{Hg}$ but none $>45 \mathrm{~mm} \mathrm{Hg}$; (3) none $<35 \mathrm{~mm} \mathrm{Hg}$ but some $>45 \mathrm{~mm} \mathrm{Hg}$; and (4) some $<35 \mathrm{~mm} \mathrm{Hg}$ and some $>45 \mathrm{~mm} \mathrm{Hg}$. An investigator blinded to the autoregulation and MRI data (R.C.-V.) created a perinatal insult score to grade the birth asphyxia severity (Table 1)

\section{Autoregulation Monitoring}

Neonates received bilateral forehead NIRS monitoring with an INVOS 5100 (Medtronic, Minneapolis, MN, USA). HVx was calculated from the NIRS and arterial blood pressure signals as previously described during hypothermia, rewarming (defined as rectal temperature $34.1-36.5^{\circ} \mathrm{C}$ ), and the first $6 \mathrm{~h}$ of normothermia [3-5, 11]. Briefly, HVx was determined from deoxygenated and oxygenated hemoglobin optical densities [11], which decreases the sensitivity of this index to changes in systemic oxygenation when compared to metrics based solely on oxyhemoglobin. We synchronously sampled MAP from the arterial blood pressure catheter and NIRS signals using ICM+ software (Cambridge Enterprises, Cambridge, UK). HVx is calculated by a continuous, moving correlation coefficient between MAP and the NIRS rTHb (rTHb = 1 - optical density_A*50), a surrogate measure of cerebral blood volume [11], after removal of signal artifacts and high-frequency waves from respiration and pulse $[3-5,14]$. When autoregulatory vasoreactivity is functional, $\mathrm{rTHb}$ and MAP have negative or near-zero correlation, and HVx is negative or near zero. During periods of dysfunctional autoregulation, rTHb and MAP become positively correlated, and $\mathrm{HVx}$ approaches +1 with progressive impairments in autoregulation [11]. We verified that neonates did not have unilateral intracranial lesions and averaged the right and left $\mathrm{HVx}$ values for sorting into 5-mm $\mathrm{Hg}$ bins of MAP. The most negative $\mathrm{HVx}$ identified the $\mathrm{MAP}_{\mathrm{OPT}}$ at which autoregulation was most robust with maximal vasoreactivity to changes in MAP during hypothermia, rewarming, and normothermia. The neonate was coded as "unidentifiable MAP ${ }_{\mathrm{OPT}}$ " if a nadir in $\mathrm{HVx}$ could not be identified [3-5]. An investigator (J.K.L.) blinded to outcomes and medical histories identified the $\mathrm{MAP}_{\mathrm{OPT}}$ values with corroboration by additional investigators (F.J.N. and M.M.G.). Blood pressure was analyzed as the (1) maximal blood pressure deviation below or above $\mathrm{MAP}_{\mathrm{OPT}}$; (2) duration with blood pressure below, within, or above $\mathrm{MAP}_{\mathrm{OPT}}$ analyzed as a percentage of the autoregulation 
Table 1. Perinatal insult score for grading birth asphyxia severity

\begin{tabular}{|c|c|c|c|}
\hline Parameter & 2 points & 1 point & 0 points \\
\hline $\mathrm{pH}$ from the umbilical cord or arterial blood gas from the 1st hour of life & $\leq 7.00$ & 7.01 to $\leq 7.15$ & $>7.15$ \\
\hline Base deficit from the umbilical cord or arterial blood gas from the 1 st hour of life & $\leq-16$ & -15 to $\leq-10$ & $>-10$ \\
\hline Sarnat stage & 3 & 2 & 1 \\
\hline Apgar score at $10 \mathrm{~min}^{\mathrm{b}}$ & & $\leq 5$ & $>5$ \\
\hline Mechanical ventilation ${ }^{\mathrm{b}}$ & & Yes & No \\
\hline Emergency delivery & & Yes & No \\
\hline
\end{tabular}

a The perinatal insult score ranges from 1 (mild perinatal event) to 8 (severe perinatal event) and measures the severity of birth asphyxia. ${ }^{b}$ If the Apgar score was not available, the baby received 1 point if mechanical ventilation was provided.

monitoring period; and (3) area under the curve (AUC; $\min \times \mathrm{mm}$ $\mathrm{Hg} / \mathrm{h}$ ) to combine time ( $\mathrm{min}$ ) spent with blood pressure below $\mathrm{MAP}_{\mathrm{OPT}}$ and blood pressure deviation $\left(\mathrm{mm} \mathrm{Hg}\right.$ ) below MAP $\mathrm{OPT}_{\mathrm{OPT}}$ normalized for the monitoring duration $(h)$ in each period $[4,15]$. We also calculated the percentage of the hypothermia, rewarming, and normothermia periods that neonates spent with MAP below gestational age in weeks $+5 \mathrm{~mm} \mathrm{Hg}$, a common clinical guide for neonatal hemodynamic goals [16].

\section{Brain MRI}

Neonates received brain MRIs after completing hypothermia and autoregulation monitoring according to our published protocol [3]. MRI studies were performed on a 1.5-T clinical scanner (Avanto; Siemens, Erlangen, Germany) by using a standard neonatal 8-channel head coil under natural sleep (without general anesthesia). Standard neonatal brain MRI with sagittal T1-weighted, axial T2-weighted, and axial susceptibility-weighted imaging was obtained. A single-shot, spin echo, echo planar axial diffusion tensor imaging sequence with diffusion gradients along 20 noncollinear directions was acquired. Trace of diffusion images and apparent diffusion coefficient (ADC) maps were automatically calculated by the vendor-specific software in the MRI scanner. Image analysis was performed on the PACS (picture archiving and communication system) workstation [4]. Two experienced pediatric neuroradiologists (A.T. and T.A.G.M.H.) categorized the brain injury as none, mild, moderate, or severe [3] in the paracentral gyri, white matter, posterior limb of the internal capsule (PLIC), putamen and globus pallidus, thalamus, and brain stem. These regions are associated with adverse neurologic outcomes from HIE [1721]. The radiologists were blinded to the neonates' $\mathrm{HVx}$, blood pressures, and clinical history.

\section{Statistical Analysis}

Data were analyzed with Rv3.2 (Vienna, Austria) and presented as means with standard deviations (SD) or medians with interquartile ranges as appropriate. Associations between brain injury on MRI and the blood pressure autoregulation parameters were analyzed separately for each period (hypothermia, rewarming, or normothermia). Classifications of regional brain injury on MRI were analyzed for their associations with the predictors $\left(\mathrm{MAP}_{\mathrm{OPT}}\right.$; blood pressure in relation to $\mathrm{MAP}_{\mathrm{OPT}}$ [the percentage of time spent with MAP below, within, or above MAP ${ }_{\mathrm{OPT}}$; the maximal MAP deviation above or below $\mathrm{MAP}_{\mathrm{OPT}}$; and the AUC of MAP below
$\mathrm{MAP}_{\mathrm{OPT}}$ in each period]; the percentage of time spent with MAP below gestational age $+5 \mathrm{~mm} \mathrm{Hg}$ in each period; and the mean $\mathrm{rSO}_{2}$ averaged between the right and left sides across each period) with ordered polytomous regression for proportional increase in injury [22]. Each analysis was adjusted for $\mathrm{PaCO}_{2}$ category, presence of seizures, vasopressor treatment, and perinatal insult score. These covariates were selected due to their potential influence on cerebral autoregulation and neurologic injury [6-8, 23-25]. Relationships between perinatal insult score and brain injury were estimated with polytomous regression for ordered categorical outcomes. Finally, we examined the neonates' blood pressures in relation to $\mathrm{MAP}_{\mathrm{OPT}}$ as they progressed through hypothermia, rewarming, and normothermia using a Pearson's correlation coefficient to compare the percentages of the autoregulation monitoring period with blood pressure below, within, or above MAP ${ }_{\mathrm{OPT}}$ between hypothermia versus rewarming; hypothermia versus normothermia; and rewarming versus normothermia.

\section{Results}

We screened 122 newborns with HIE. Forty-seven (39\%) were ineligible for the study because of an unreliable arterial catheter blood pressure tracing (16), parents' refusal to consent to the study (9), transfer for ECMO (6), death before initiation of HVx monitoring (5), technical difficulties (5), inadequate resources (3), complex heart disease (1), coagulopathy (1), or parents' inability to speak English or Spanish (1). Therefore, 75 (61\%) neonates with HIE were monitored with $\mathrm{HVx}$. Eleven did not have diagnostic brain MRI for evaluation because of motion artifacts (7) or withdrawal of support before MRI acquisition (4).

The final sample size was 64 neonates (38 boys, 26 girls), and data were analyzed among those with an identifiable $\mathrm{MAP}_{\mathrm{OPT}}$ (see below). All 64 neonates with brain MRI data had $\mathrm{HVx}$ monitoring during hypothermia (mean duration $46.5 \mathrm{~h}$ [SD, 19.8]), 59 during rewarming 


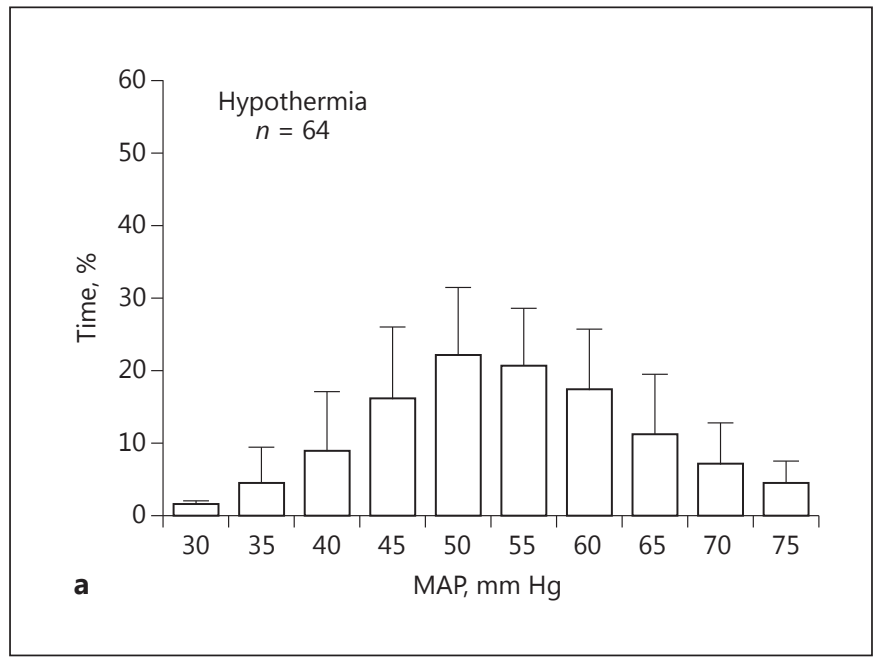

Fig. 1. The distribution of neonates' mean arterial blood pressure (MAP) during hypothermia (a), rewarming (b), and normothermia (c). Means with SD are shown.

(duration $6.3 \mathrm{~h}[\mathrm{SD}, 2.6]$ ), and 57 during normothermia (duration $5.3 \mathrm{~h}[\mathrm{SD}, 1.6]$ ). HVx monitoring was stopped after hypothermia in 2 neonates because of technical problems and in 3 who were transferred to another unit; these 3 neonates underwent the therapeutic hypothermia protocol, did not receive ECMO, and had brain MRI data analyzed in the study. Two neonates had HVx monitoring stopped after rewarming because of early removal of the NIRS (1) or arterial blood pressure catheter (1). Patient descriptions are listed in Table 2. The hemoglobin levels were $15.5 \mathrm{~g} / \mathrm{dL}$ (SD, 2.0) during HVx monitoring. Twelve neonates received hydrocortisone.

The neonates' blood pressure distributions are shown in Figure 1 . We identified MAP ${ }_{\mathrm{OPT}}$ values in 55/64 neonates (86\%; 32 boys, 23 girls) during hypothermia, 54/59 (92\%; 31 boys, 23 girls) during rewarming, and 55/57 (97\%; 35

Optimizing Autoregulation for Neonatal Brain Hypoxia
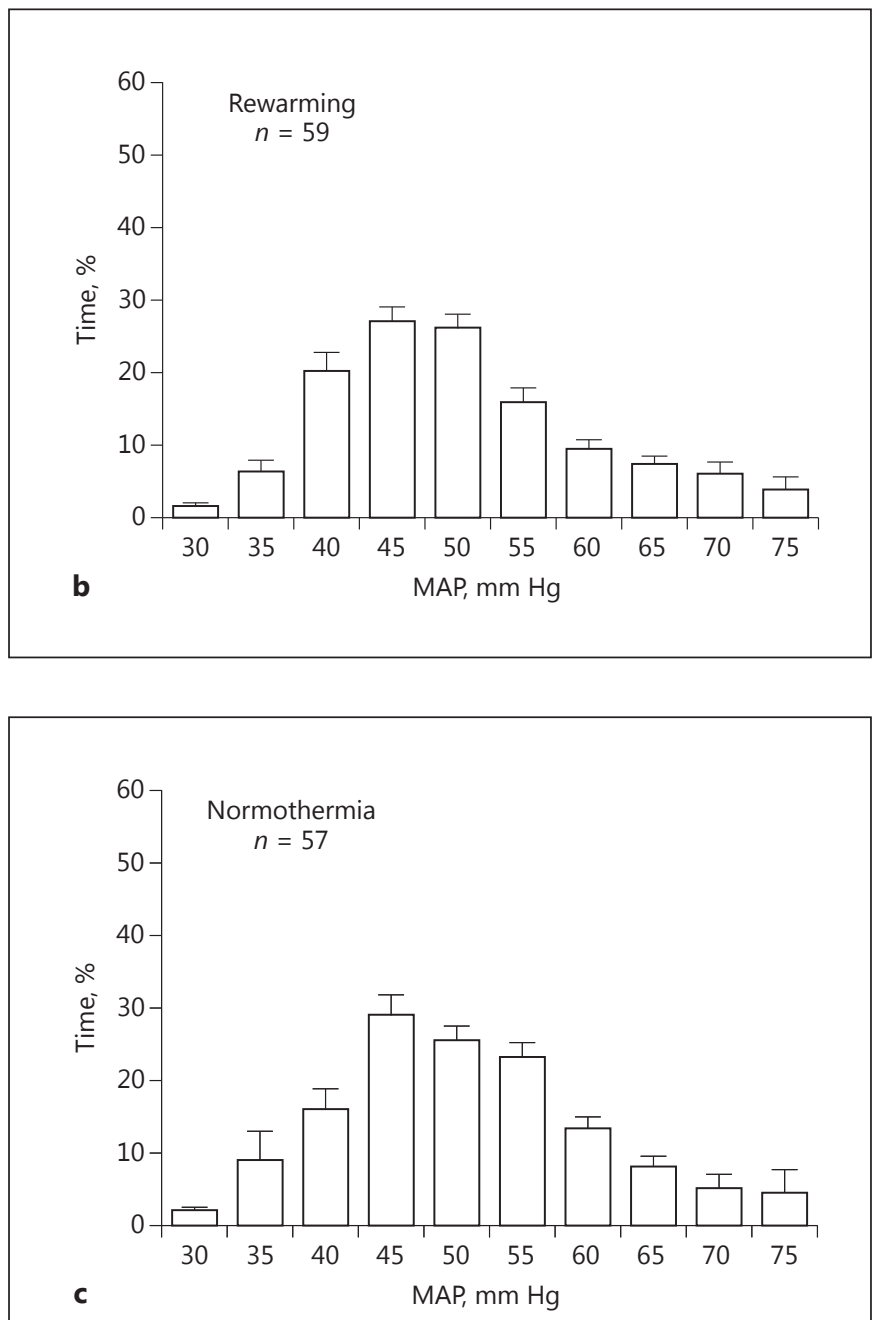

Table 2. Neonatal parameters that may influence blood pressure autoregulation (64 neonates) ${ }^{\mathrm{a}}$

\begin{tabular}{lc}
\hline Vasopressor (any), $n(\%)$ & $41(64)$ \\
Seizures (any), $n(\%)$ & $24(38)$ \\
PaCO $_{2}, n(\%)$ & \\
All $(35-45 \mathrm{~mm} \mathrm{Hg})$ & $6(10)$ \\
Some $<35 \mathrm{~mm} \mathrm{Hg}$, all $<45 \mathrm{~mm} \mathrm{Hg}$ & $11(17)$ \\
None $<35 \mathrm{~mm} \mathrm{Hg}$, some $>45 \mathrm{~mm} \mathrm{Hg}$ & $29(45)$ \\
Some $<35 \mathrm{~mm} \mathrm{Hg}$, some $>45 \mathrm{~mm} \mathrm{Hg}$ & $18(28)$ \\
Median perinatal insult score (IQR) & $6(5-7)$ \\
\hline
\end{tabular}

$n$ (\%) of neonates and median (interquartile range, IQR). a These covariates were adjusted for in the analysis.

Dev Neurosci 2017;39:248-256 


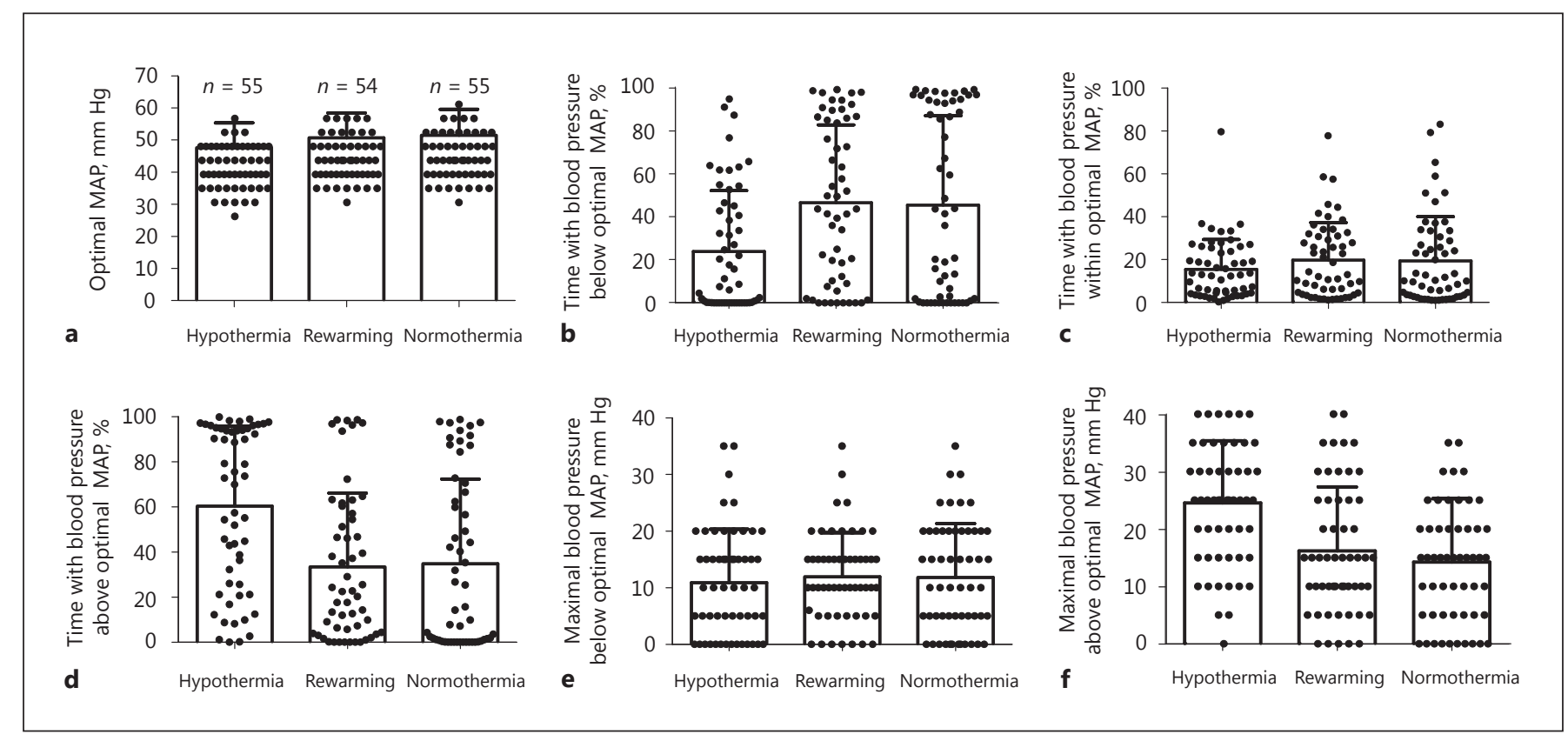

Fig. 2. The neonates' optimal mean arterial blood pressure (MAP) values (a), the percentage of each period spent with blood pressure below (b), within $(\mathbf{c})$, or above optimal MAP (d), and the maximal blood pressure deviation below (e) or above optimal MAP (f). Means with SD are shown. Each circle represents 1 neonate.

boys, 20 girls) during normothermia. The mean $\mathrm{MAP}_{\mathrm{OPT}}$ values were $50 \mathrm{~mm} \mathrm{Hg}(\mathrm{SD}, 10)$ in each period. (Fig. 2a). The neonates who were coded as having an unidentifiable $\mathrm{MAP}_{\mathrm{OPT}}$ did not display a clear HVx nadir in the bar graph of MAP versus HVx. The neonates' blood pressures in relation to $\mathrm{MAP}_{\mathrm{OPT}}$ are shown in Figures $2 \mathrm{~b}-\mathrm{f}$.

We also compared neonates who did not have brain MRI (and were excluded from the study) with those who did. The perinatal injury scores among neonates who did or did not receive an MRI were 6 (SD, 1.3) and 6 (SD, $1.5)$, respectively. The MAP ${ }_{\text {OPT }}$ values between neonates with or without MRI were $50 \mathrm{~mm} \mathrm{Hg}(\mathrm{SD}, 10 ; n=55)$ and $45 \mathrm{~mm} \mathrm{Hg}(\mathrm{SD}, 10 ; n=10)$ during hypothermia $(p>0.10)$ and $50 \mathrm{~mm} \mathrm{Hg}(\mathrm{SD}, 10 ; n=54)$ and $45 \mathrm{~mm}$ $\mathrm{Hg}(\mathrm{SD}, 10 ; n=4)$ during rewarming $(p>0.10)$, respectively. However, $\mathrm{MAP}_{\mathrm{OPT}}$ during normothermia was lower in neonates without MRI (mean, $40 \mathrm{~mm} \mathrm{Hg}$; SD, $10 ; n=5$ ) than in those with MRI (mean, $50 \mathrm{~mm} \mathrm{Hg}$; $\mathrm{SD}, 10 ; n=55 ; p=0.026$ ). Blood pressure in relation to $\mathrm{MAP}_{\mathrm{OPT}}$ during hypothermia and rewarming was similar between neonates with and without MRI ( $p>0.05$; data not shown). Neonates without MRI had greater duration $(p=0.018)$ and deviation $(p=0.017)$ in blood pressure above $\mathrm{MAP}_{\mathrm{OPT}}$ during normothermia than neonates with MRIs.
Table 3. Distribution of categorical brain injuries among the 64 neonates

\begin{tabular}{llccc}
\hline \multirow{2}{*}{ Region } & \multicolumn{4}{l}{ Injury, $n(\%)$} \\
\cline { 2 - 5 } & none & mild & moderate & severe \\
\hline Central gyrus & $37(58)$ & $14(22)$ & $6(9)$ & $7(11)$ \\
White matter & $13(20)$ & $28(44)$ & $10(16)$ & $13(20)$ \\
Basal ganglia & $28(44)$ & $19(30)$ & $10(15)$ & $7(11)$ \\
Thalamus & $27(42)$ & $17(27)$ & $11(17)$ & $9(14)$ \\
PLIC & $42(66)$ & $11(17)$ & $6(9)$ & $5(8)$ \\
Brain stem & $28(43)$ & $19(30)$ & $10(16)$ & $7(11)$ \\
\hline
\end{tabular}

PLIC, posterior limb of the internal capsule.

\section{Autoregulation and Brain Injury on MRI}

MRIs were obtained at 8.5 days of life (SD, 2.5; range, 4-16), and brain injury was graded in all 64 neonates (Table 3). The perinatal insult score was not associated with injury in the white matter, PLIC, putamen and globus pallidus, thalamus, or brain stem $(p>0.05 ; n=64)$. However, more severe perinatal insult was related to greater injury in the paracentral gyri $(p=0.009 ; n=64)$.

In the analysis adjusted for $\mathrm{PaCO}_{2}$, seizures, vasopressors, and perinatal insult severity, greater AUC below 
$\mathrm{MAP}_{\mathrm{OPT}}$ during rewarming $(\beta=0.002 ; p=0.047, n=54)$ was associated with more severe injury in the white matter. More time with blood pressure within the $5-\mathrm{mm} \mathrm{Hg}$ range of $\mathrm{MAP}_{\mathrm{OPT}}$ during rewarming was related to less white matter injury $(\beta=-0.044, p=0.017, n=54)$ (online suppl. Table 1; for all online suppl. material, see www. karger.com/doi/10.1159/000452833). The autoregulatory parameters were not associated with PLIC injury ( $p>$ 0.05 for all comparisons; data not shown).

Both perinatal insult severity $(p=0.009 ; n=64)$ and blood pressure affected paracentral gyrus injury. The analyses, therefore, included adjustments for perinatal insult severity. Among neonates with an identified MAP $_{\mathrm{OPT}}$, greater duration $(\beta=0.041 ; p=0.007 ; n=55)$, deviation $(\beta=0.104 ; p=0.012 ; n=55)$, and AUC $(\beta=$ $0.005 ; p=0.020 ; n=55)$ of blood pressure below $\mathrm{MAP}_{\mathrm{OPT}}$ during hypothermia correlated with more severe injury in the paracentral gyri. In addition, greater duration $(\beta=$ $-0.027, p=0.020, n=55)$ and deviation $(\beta=-0.069, p=$ $0.038, n=55)$ of blood pressure above $\mathrm{MAP}_{\mathrm{OPT}}$ during hypothermia was related to decreased paracentral gyrus injury (online suppl. Table 2).

Spending more time with blood pressure within $\mathrm{MAP}_{\mathrm{OPT}}$ during normothermia was associated with less injury in putamen and globus pallidus $(\beta=-0.034, p=$ $0.040, n=55$ ) (online suppl. Table 3 ). Finally, greater duration of blood pressure within $\mathrm{MAP}_{\mathrm{OPT}}$ during normothermia was related to less brain stem injury $(\beta=-0.035$, $p=0.027, n=55$ ) (online suppl. Table 4 ). Blood pressure relative to $\mathrm{MAP}_{\mathrm{OPT}}$ was not associated with thalamic injury ( $p>0.05$ for all comparisons; data not shown).

\section{$\mathrm{rSO}_{2}$ and Blood Pressure Threshold $5 \mathrm{~mm} \mathrm{Hg}$ above \\ Gestational Age}

In the adjusted analysis of all neonates with an identified $\mathrm{MAP}_{\mathrm{OPT}}, \mathrm{rSO}_{2}$ was unrelated to brain injury in any anatomic region ( $p>0.05$; data not shown). Moreover, the duration of blood pressure below the gestational age $+5 \mathrm{~mm} \mathrm{Hg}$ in any period was not associated with injury in any brain region in the adjusted analysis of neonates with an identified $\mathrm{MAP}_{\mathrm{OPT}}(p>0.05$; data not shown).

\section{Blood Pressure in Relation to MAP ${ }_{O P T}$ during}

Progression from Hypothermia to Normothermia

Neonates with greater duration of blood pressure below $\mathrm{MAP}_{\mathrm{OPT}}$ during hypothermia also spent more time with blood pressure below $\mathrm{MAP}_{\mathrm{OPT}}$ during rewarming $(r=0.49 ; p<0.001)$. The durations of blood pressure below $\mathrm{MAP}_{\mathrm{OPT}}$ were not correlated between hypothermia and normothermia $(r=0.20 ; p=0.17)$ or between re-

Optimizing Autoregulation for Neonatal Brain Hypoxia warming and normothermia $(r=0.20 ; p=0.16)$. Neonates who spent more time during hypothermia with blood pressure above $\mathrm{MAP}_{\mathrm{OPT}}$ also spent more time with blood pressure above $\mathrm{MAP}_{\mathrm{OPT}}$ during rewarming $(r=$ $0.50, p<0.001)$. Time with blood pressure above $\mathrm{MAP}_{\mathrm{OPT}}$ was not correlated between hypothermia and normothermia $(r=0.21 ; p=0.15)$ or rewarming versus normothermia $(r=0.22 ; p=0.12)$. Finally, neonates with greater duration of blood pressure within $\mathrm{MAP}_{\mathrm{OPT}}$ during hypothermia also spent more time with blood pressure within $\mathrm{MAP}_{\mathrm{OPT}}$ during rewarming $(r=0.40 ; p=0.007)$ and normothermia $(r=0.32 ; p=0.025)$. Time with blood pressure within $\mathrm{MAP}_{\mathrm{OPT}}$ during rewarming and normothermia were not correlated $(r=0.16 ; p=0.273)$.

\section{Discussion}

We provide evidence that blood pressure deviations from the range of optimal autoregulation during and after therapeutic hypothermia are associated with brain injury on MRI in neonates with HIE, but this relationship is complex and varies by anatomic region. Brain injury was not affected by perinatal insult severity in most regions; thus, blood pressures that do not optimize autoregulation during and after therapeutic hypothermia independently affect subsequent brain injury measurements on MRI. Greater duration and deviation of blood pressure below $\mathrm{MAP}_{\mathrm{OPT}}$ were associated with greater injury in the white matter and paracentral gyri. Blood pressure within MAP $_{\mathrm{OPT}}$ related to lesser injury in the white matter, putamen and globus pallidus, and brain stem. Hence, hemodynamic management to optimize autoregulation using $\mathrm{HVx}$ could serve as a therapeutic adjunct to hypothermia in HIE.

Brain MRI is a biomarker of neurodevelopmental outcome in HIE [26, 27]. We used T1/T2-weighted images, trace of diffusion images, and ADC maps given the high specificity and sensitivity of these sequences in predicting neurodevelopmental outcomes [28]. In addition, qualitatively scoring brain injury on MRI during the first 2 weeks of life and after therapeutic hypothermia has consistent and high predictive accuracy for neurodevelopmental outcome [29]. Signal abnormalities in conventional MRI, trace of diffusion images, and ADC maps in the cortex, PLIC, white matter, putamen and globus pallidus, thalamus, and brain stem predict disability or death after HIE $[9,18-21]$. Thus, identifying methods to reduce brain injury on MRI may improve neurodevelopmental outcomes.

Dev Neurosci 2017;39:248-256

DOI: $10.1159 / 000452833$
253 
The potential of targeting the autoregulatory blood pressure range as a therapeutic adjunct to hypothermia is unclear. Hypothermia after hypoxic-ischemic brain injury is known to decrease cerebral blood flow [30, 31], although the blood pressure limits of autoregulation and $\mathrm{MAP}_{\mathrm{OPT}}$ can still be determined during hypothermia [3, $14,31]$. Severe birth asphyxia may cause brain damage with dysfunctional autoregulation and/or hemodynamic instability. Alternatively, some neonates' outcomes might improve if their blood pressure is maintained to support autoregulation. We developed a perinatal insult severity score to account for the effects of birth asphyxia on autoregulation and subsequent brain injury. Reliable and accurate risk stratification methods to differentiate neonates with favorable or unfavorable neurologic outcomes are not available in HIE, although the 10-min Apgar score may be associated with neurocognitive outcomes at 5-6 years of age [32]. The perinatal insult score, which is derived from common clinical perinatal parameters, did not relate to brain injury in any region except for paracentral gyri. New and noninvasive methods that can identify neonates who are at high risk of permanent neurologic injury soon after birth are urgently needed. These high-risk babies may benefit from adjuvant treatments, such as methods that support autoregulatory function. Alternatively, identifying neonates with poor autoregulation using HVx may indicate those at highest risk of neurologic injury on MRI. Nonetheless, the fact that the perinatal insult score was not associated with brain injury in most regions suggests blood pressures that do not support autoregulation during the first 4 days of life may independently relate to injury evolution in the white matter, putamen and globus pallidus, and brain stem. Such an independent relationship does not strictly distinguish whether blood pressure deviation from $\mathrm{MAP}_{\mathrm{OPT}}$ causes additional injury or if the greater injury, particularly in the brain stem, produces greater blood pressure lability.

Blood pressure deviation from $\mathrm{MAP}_{\mathrm{OPT}}$ measured by frontal NIRS, which predominantly measures the frontal cortex, related to injury in regions not captured by NIRS, including other cortical (i.e. paracentral gyri) and noncortical regions. Autoregulation involves large cerebral arteries and pial arterioles, and thus has a prominent macrocirculatory component upstream of local parenchymal tissue. Much of the autoregulatory vasoactivity is attributed to the intrinsic myogenic response to transmural pressure. Thus, large regional differences in $\mathrm{MAP}_{\mathrm{OPT}}$ are not expected after global cerebral ischemia, and large and prolonged deviations of blood pressure from $\mathrm{MAP}_{\mathrm{OPT}}$ measured in the frontal lobe will likely affect perfusion throughout much of the brain. While more precise methods for determining regional autoregulatory properties would be ideal, using $\mathrm{MAP}_{\mathrm{OPT}}$ from the frontal lobe is a reasonable first approximation for targeting hemodynamic management.

We identified associations between the autoregulation parameters and brain injury during hypothermia (paracentral gyrus injury), rewarming (white matter injury), and normothermia (putamen and globus pallidus and brain stem injuries). Larger studies are needed to define the interactions between temperature and autoregulation in specific regions. Hypothermia and rewarming do not fully protect cortical gray and white matter from apoptotic cell death [33-35], and blood pressure below the optimal autoregulatory level may render these regions more vulnerable to cell death. By contrast, the neuroprotection afforded by hypothermia in the putamen [36] may delay vulnerability to suboptimal autoregulatory function until normothermia.

Blood pressure within $\mathrm{MAP}_{\mathrm{OPT}}$ was associated with lesser injury in the white matter, putamen and globus pallidus, and brain stem. Blood pressure above $\mathrm{MAP}_{\mathrm{OPT}}$ was related to lesser injury in paracentral gyri. Although it is tempting to speculate that targeting or exceeding $\mathrm{MAP}_{\text {OPT }}$ would confer neuroprotection, caution must be exercised when raising blood pressure to not induce cardiopulmonary injury with increases in afterload, intravascular volume, and cardiogenic strain. Nonetheless, identifying $\mathrm{MAP}_{\mathrm{OPT}}$ related better to brain injury than did $\mathrm{rSO}_{2}$ or blood pressure based on gestational age +5 $\mathrm{mm} \mathrm{Hg}$. Larger studies across multiple institutions are needed to further explore whether hemodynamic management that targets $\mathrm{MAP}_{\mathrm{OPT}}$ reduces neurologic injury compared to conventional neonatal clinical guidelines, including those based on the gestational age [16].

Neonates who spent a greater duration of time with blood pressure below $\mathrm{MAP}_{\mathrm{OPT}}$ during hypothermia also spent more of rewarming with blood pressure below $\mathrm{MAP}_{\mathrm{OPT}}$. Conversely, neonates with greater duration of blood pressure above $\mathrm{MAP}_{\mathrm{OPT}}$ during hypothermia also had more time with blood pressure above $\mathrm{MAP}_{\mathrm{OPT}}$ during rewarming. The neonates' time with blood pressure within the 5-mm $\mathrm{Hg}$ bin of $\mathrm{MAP}_{\mathrm{OPT}}$ were also correlated across hypothermia, rewarming, and normothermia. This consistent relationship between blood pressure and $\mathrm{MAP}_{\mathrm{OPT}}$ across time and temperature phases emphasizes the importance of using continuous HVx monitoring to identify neonates with suboptimal autoregulation early in hypothermia because these neonates are at high risk of continued suboptimal autoregulation during rewarming and normothermia. 
Causal relationships were not addressed by this observational study. Because of the small sample size, the results may underidentify associations between injury and autoregulation in this single-center study. The exclusion of neonates who received ECMO or had withdrawal of care and did not receive HVx monitoring or brain MRIs creates selection bias. Neonates excluded from the study because they lacked MRI data were similar in perinatal insult score and autoregulation data to those included in the study with the exception of $\mathrm{MAP}_{\mathrm{OPT}}$ during normothermia; 5 neonates without MRI had lower MAP ${ }_{\mathrm{OPT}}$ values and greater blood pressure deviation above $\mathrm{MAP}_{\mathrm{OPT}}$ during normothermia than the 55 neonates who received MRI. HVx monitoring could only begin after an arterial blood pressure cannula was established; therefore, early autoregulatory instability may not have been captured. We analyzed the data as a percentage of the autoregulation monitoring period and normalized the AUC for the monitoring duration to account for different monitoring durations in hypothermia. We monitored HVx more consistently during rewarming and normothermia. Steroid use and delays in initiating hypothermia may affect blood pressure and neurologic injury, and we did not adjust for these potential confounders.

\section{Conclusions}

NIRS-derived $\mathrm{HVx}$ autoregulation monitoring can identify the blood pressure range with most robust autoregulatory vasoreactivity in neonates who receive thera- peutic hypothermia for HIE. The relationship between cerebrovascular autoregulation and MRI-documented neurologic injury in HIE patients is complex, may vary by anatomic region, and is largely independent of the perinatal injury insult severity. Determination of $\mathrm{MAP}_{\mathrm{OPT}}$ that supports maximal cerebrovascular reactivity to perturbations in MAP may identify hemodynamic ranges that limit the progression of neural injury in several regions. The influence of hypothermia and rewarming on the effects of autoregulatory hemodynamics and brain injury requires further study.

\section{Acknowledgments}

We are grateful to Claire Levine, MS, ELS, for her editorial assistance.

\section{Funding Sources}

Support was provided by NIH R01HD070996, R01HD086058, and R01HD074593 (F.J.N.); K08NS080984 and R21HD072845 (J.K.L.); R01NS060703 (R.C.K.); Johns Hopkins University Clinician Scientist Award and American Heart Association Grant-inAid (J.K.L.); and the Sutland-Pakula Endowment for Neonatal Research (R.C.-V.).

\section{Disclosure Statement}

Drs. Lee, Northington, Gilmore, and Chavez-Valdez received research support from Medtronic for a separate study.

\section{References}

1 Shankaran S, Pappas A, McDonald SA, et al: Childhood outcomes after hypothermia for neonatal encephalopathy. N Engl J Med 2012; 366:2085-2092.

2 Azzopardi D, Strohm B, Marlow N, et al: Effects of hypothermia for perinatal asphyxia on childhood outcomes. N Engl J Med 2014;371: 140-149.

3 Howlett JA, Northington FJ, Gilmore MM, et al: Cerebrovascular autoregulation and neurologic injury in neonatal hypoxic-ischemic encephalopathy. Pediatr Res 2013;74:525535.

4 Tekes A, Poretti A, Scheurkogel MM, et al: Apparent diffusion coefficient scalars correlate with near-infrared spectroscopy markers of cerebrovascular autoregulation in neonates cooled for perinatal hypoxic-ischemic injury. AJNR Am J Neuroradiol 2015;36:188-193.
5 Burton VJ, Gerner G, Cristofalo E, et al: A pilot cohort study of cerebral autoregulation and 2-year neurodevelopmental outcomes in neonates with hypoxic-ischemic encephalopathy who received therapeutic hypothermia. BMC Neurol 2015;15:209.

6 Pollock JM, Deibler AR, Whitlow CT, et al: Hypercapnia-induced cerebral hyperperfusion: an underrecognized clinical entity. AJNR Am J Neuroradiol 2009;30:378-385.

7 Armstead WM, Riley J, Vavilala MS: Dopamine prevents impairment of autoregulation after traumatic brain injury in the newborn pig through inhibition of up-regulation of endothelin-1 and extracellular signal-regulated kinase mitogen-activated protein kinase. Pediatr Crit Care Med 2013; 14:e103-e111.
8 Armstead WM, Kiessling JW, Riley J, Kofke WA, Vavilala MS: Phenylephrine infusion prevents impairment of ATP- and calciumsensitive potassium channel-mediated cerebrovasodilation after brain injury in female, but aggravates impairment in male, piglets through modulation of ERK MAPK upregulation. J Neurotrauma 2011;28:105-111.

9 Massaro AN, Govindan RB, Vezina G, et al: Impaired cerebral autoregulation and brain injury in newborns with hypoxic-ischemic encephalopathy treated with hypothermia. J Neurophysiol 2015;114:818-824.

10 Tian F, Tarumi T, Liu H, Zhang R, Chalak L: Wavelet coherence analysis of dynamic cerebral autoregulation in neonatal hypoxic-ischemic encephalopathy. Neuroimage Clin 2016; 11:124-132. 
11 Lee JK, Kibler KK, Benni PB, et al: Cerebrovascular reactivity measured by near-infrared spectroscopy. Stroke 2009;40:1820-1826.

12 Shankaran S, Laptook AR, Ehrenkranz RA, et al: Whole-body hypothermia for neonates with hypoxic-ischemic encephalopathy. N Engl J Med 2005;353:1574-1584.

13 Pappas A, Shankaran S, Laptook AR, et al: Hypocarbia and adverse outcome in neonatal hypoxic-ischemic encephalopathy. J Pediatr 2011;158:752.e1-758.e1.

14 Larson AC, Jamrogowicz JL, Kulikowicz E, et al: Cerebrovascular autoregulation after rewarming from hypothermia in a neonatal swine model of asphyxic brain injury. J Appl Physiol (1985) 2013;115:1433-1442.

15 Lee JK, Brady KM, Chung SE, et al: A pilot study of cerebrovascular reactivity autoregulation after pediatric cardiac arrest. Resuscitation 2014;85:1387-1393.

16 Gretchen CB, Rayannavar AS: Cardiology; in Engorn B, Flerlage J (eds): The Harriet Lane Handbook, ed 20. Philadelphia, Saunders, Elsevier, 2015, pp 127-171.

17 Martinez-Biarge M, Diez-Sebastian J, Wusthoff CJ, et al: Feeding and communication impairments in infants with central grey matter lesions following perinatal hypoxic-ischaemic injury. Eur J Paediatr Neurol 2012;16: 688-696.

18 Rutherford M, Ramenghi LA, Edwards AD, et al: Assessment of brain tissue injury after moderate hypothermia in neonates with hypoxic-ischaemic encephalopathy: a nested substudy of a randomised controlled trial. Lancet Neurol 2010;9:39-45.

19 van Schie PE, Schijns J, Becher JG, Barkhof F, van Weissenbruch MM, Vermeulen RJ: Longterm motor and behavioral outcome after perinatal hypoxic-ischemic encephalopathy. Eur J Paediatr Neurol 2015;19:354-359.
20 Cavalleri F, Lugli L, Pugliese M, et al: Prognostic value of diffusion-weighted imaging summation scores or apparent diffusion coefficient maps in newborns with hypoxic-ischemic encephalopathy. Pediatr Radiol 2014;44: 1141-1154.

21 Goergen SK, Ang H, Wong F, et al: Early MRI in term infants with perinatal hypoxic-ischaemic brain injury: interobserver agreement and MRI predictors of outcome at 2 years. Clin Radiol 2014;69:72-81.

22 Brant R: Assessing proportionality in the proportional odds model for ordinal logistic regression. Biometrics 1990;46:1171-1178.

23 Monrad P, Sannagowdara K, Bozarth X, et al: Haemodynamic response associated with both ictal and interictal epileptiform activity using simultaneous video electroencephalography/near infrared spectroscopy in a withinsubject study. J Near Infrared Spectrosc 2015; 23:209-218.

24 Lingappan K, Kaiser JR, Srinivasan C, Gunn AJ: Relationship between $\mathrm{PCO}_{2}$ and unfavorable outcome in infants with moderate-to-severe hypoxic ischemic encephalopathy. Pediatr Res 2016;80:204-208.

25 Massaro AN, Murthy K, Zaniletti I, et al: Short-term outcomes after perinatal hypoxic ischemic encephalopathy: a report from the Children's Hospitals Neonatal Consortium HIE focus group. J Perinatol 2015;35:290296.

26 Nanavati T, Seemaladinne N, Regier M, Yossuck P, Pergami P: Can we predict functional outcome in neonates with hypoxic ischemic encephalopathy by the combination of neuroimaging and electroencephalography? Pediatr Neonatol 2015;56:307-316.

27 Massaro AN: MRI for neurodevelopmental prognostication in the high-risk term infant. Semin Perinatol 2015;39:159-167.

28 van Laerhoven $H$, de Haan TR, Offringa M, Post B, van der Lee JH: Prognostic tests in term neonates with hypoxic-ischemic encephalopathy: a systematic review. Pediatrics 2013;131:88-98.
29 Skranes JH, Cowan FM, Stiris T, Fugelseth D, Thoresen M, Server A: Brain imaging in cooled encephalopathic neonates does not differ between four and 11 days after birth. Acta Paediatr 2015;104:752-758.

30 Buckley EM, Patel SD, Miller BF, Franceschini MA, Vannucci SJ: In vivo monitoring of cerebral hemodynamics in the immature rat: effects of hypoxia-ischemia and hypothermia. Dev Neurosci 2015;37:407-416.

31 Lee JK, Brady KM, Mytar JO, et al: Cerebral blood flow and cerebrovascular autoregulation in a swine model of pediatric cardiac arrest and hypothermia. Crit Care Med 2011;39: 2337-2345.

32 Natarajan G, Shankaran S, Laptook AR, et al: Apgar scores at $10 \mathrm{~min}$ and outcomes at 6-7 years following hypoxic-ischaemic encephalopathy. Arch Dis Child Fetal Neonatal Ed 2013;98:F473-F479.

33 Wang B, Armstrong JS, Reyes M, et al: White matter apoptosis is increased by delayed hypothermia and rewarming in a neonatal piglet model of hypoxic ischemic encephalopathy. Neuroscience 2016;316:296-310.

34 Wang B, Armstrong JS, Lee JH, et al: Rewarming from therapeutic hypothermia induces cortical neuron apoptosis in a swine model of neonatal hypoxic-ischemic encephalopathy. J Cereb Blood Flow Metab 2015;35:781-793.

35 Lee JK, Wang B, Reyes M, et al: Hypothermia and rewarming activate a macroglial unfolded protein response independent of hypoxicischemic brain injury in neonatal piglets. Dev Neurosci 2016;38:277-294.

36 Mueller-Burke D, Koehler RC, Martin LJ: Rapid NMDA receptor phosphorylation and oxidative stress precede striatal neurodegeneration after hypoxic ischemia in newborn piglets and are attenuated with hypothermia. Int J Dev Neurosci 2008;26:67-76. 